\title{
A INFECÇÃo hOSPITALAR NO CONTEXTO dAS POLÍticAs RELATIVAS À SAÚDE EM SANTA CATARINA
}

Ruvani Fernandes da Silva ${ }^{1}$

Silva RF. A infecção hospitalar no contexto das políticas relativas à saúde em Santa Catarina. Rev Latino-am Enfermagem 2003 janeiro-fevereiro; 11(1):108-14.

O Ministério da Saúde tem norteado as ações de saúde no sentido de controlar e/ou melhorar a assistência. Em 1994, foi realizado, simultaneamente, estudo e pesquisa nacional para conhecer e avaliar a qualidade das ações em controle de infecção hospitalar. Os resultados apontam incidência média de infecção hospitalar 15,5\% e freqüência de hospitais que executam procedimentos de risco corretamente $42,5 \%$. O objetivo deste estudo é fazer relato da situação das comissões de controle de infecção hospitalar no contexto das políticas de saúde em Santa Catarina. O Programa de Controle de Infecção Hospitalar foi criado em 1992, tendo grande receptividade, sendo implantadas comissões em 90 hospitais, representando aumento significativo (7\% em 1992, para 43\%, em 1993). Existem, em todo o Estado, 177 comissões de controle de infecção hospitalar, totalizando 77\%. Atualmente, são 16 hospitais acreditados em controle de infecção e 30 em preparação para avaliação da acreditação.

DESCRITORES: infecção hospitalar, política de saúde, acreditação

\section{HOSPITAL INFECTION IN THE CONTEXT OF THE HEALTH POLICIES ESTABLISHED BY THE STATE OF SANTA CATARINA}

The Ministry of Health is guiding its health actions aiming at controlling and/or improving the care. Therefore, in 1994, a national study and research was developed in order to understand and evaluate the quality of the actions related to the control of hospital infection in the country. The results showed a medium incidence of hospital infection (15,5\%) and a number of hospitals that correctly perform risk procedures (42,5\%). The purpose of this study was to report the work of the Commissions for Hospital Infection Control in the context of the health policies established in the state of Santa Catarina, Brazil. The Program for Hospital Infection Control was created in 1992 and had a high receptivity, implementing commissions in 90 hospitals what represented a significant increase (from 7\% in 1992 to 43\% in 1993). There are in the state 177 Commissions for Hospital Infection Control, totaling 77\%. Nowadays, 16 hospitals are accredited in infection control and 30 are being prepared for the accreditation evaluation.

DESCRIPTORS: hospital infection, health policy, accreditation

\section{LA INFECCIÓN HOSPITALARIA EN EL CONTEXTO DE LAS POLÍTICAS RELACIONADAS CON LA SALUD EN SANTA CATARINA}

El Ministerio de Salud, tiene orientada las acciones de salud en el sentido de controlar y/o mejorar la atención. En 1994 fue realizado simultáneamente un estudio e investigación nacional para conocer y evaluar la calidad de las acciones en el control de las infecciones hospitalarias. Los resultados muestran la incidencia promedio de las infecciones hospitalarias en un $15,5 \%$ y la frecuencia de los hospitales que ejecutan procedimientos de riesgo correctamente del $42,5 \%$. El objetivo de este estudio es hacer el relato de la situación de las comisiones de control de infección hospitalaria en el contexto de las políticas de salud en Santa Catarina. El programa de control de infección Hospitalaria fue creado en 1992 teniendo gran receptividad, siendo implantadas comisiones en 90 hospitales, representando un aumento significativo (7\% en 1992 y 43\% en 1993). Existen en todo el Estado 177 comisiones de control de infección hospitalaria, totalizando 77\%. Actualmente son 16 hospitales preparados para control de infección y 30 en preparación.

DESCRIPTORES: infección hospitalaria, política de salud, acreditación

\footnotetext{
${ }^{1}$ Doutorando do Programa de Pós Graduação em Enfermagem da Universidade Federal de Santa Catarina; Professor da Universidade do Sul de Santa Catarina e Enfermeiro do Serviço de Controle de Infecção Hospitalar do Hospital Governador Celso Ramos em Florianópolis, Santa Catarina, e-mail: ruvanis@terra.com.br ou ruvanis@uol.com.br
} 
INTRODUÇÃO

O Ministério da Saúde (MS), ao longo de sua existência, tem norteado as ações de saúde no sentido de controlar e/ou melhorar a vigilância epidemiológica e a atenção aos marginalizados.

Vem se observando, no decorrer dos tempos, mais precisamente desde a década de 70 , que o setor de saúde atravessa um período de falência, e foi nesse clima que estudos e pesquisas demonstraram e discutiram a necessidade da implantação de um sistema único de saúde.

$\mathrm{Na}$ década de 80 , o agravamento da crise financeira do país atinge vários segmentos como reflexo da política econômica recessiva implantada no país, abalando, inclusive, a área da saúde. O principal foco dessa crise é o aumento dos custos e fraudes, que é identificado com as despesas na assistência médica da Previdência. O governo recorre a várias tentativas para reorganizar o sistema nacional de saúde, e, com isso, evidencia um posicionamento diferenciado entre a Federação Brasileira dos Hospitais, por meio das denúncias de fraudes, e a Associação Brasileira de Medicina de Grupo.

Foi nesse clima que, em 1981, criou-se o Conselho Consultivo de Administração de Saúde Previdenciária (CONASP), aprovado em 1982, que tinha como meta a racionalização dos gastos e reorganização do Sistema Nacional de Saúde e da Assistência à Saúde, no âmbito da Previdência Social.

Com a $8^{\text {a }}$ Conferência Nacional de Saúde, em 1986, ocorre a grande mudança, em que se propôs a reformulação de toda a política nacional na área da saúde, por meio da chamada Reforma Sanitária. Saúde é entendida como resultado das condições de vida da pessoa, direito de todos e dever do estado.

A reforma propõe a implantação do Sistema Único de Saúde (SUS), surgindo, então, uma forma de organização dos serviços de saúde no país, na qual tanto os serviços públicos quanto os privados (por meio de convênios) fazem parte de uma mesma rede, com objetivo único de atender às necessidades de promoção, proteção e recuperação da saúde da população.

Antes da Constituição Federal de $1988^{(1)}$, saúde resumia-se apenas à assistência médica prestada. A saúde não era direito de todos os brasileiros, mas um direito exclusivo de quem tivesse carteira assinada e pagasse a previdência social - Instituto Nacional da Previdência Social (INPS). Quem não estivesse nessa parcela da população pagaria as contas (minoria) ou seria atendido como indigente (maioria).

Com a universalização do direito à saúde, o Estado é responsabilizado como o seu provedor. No momento em que esse conceito é incorporado à prática, surge uma nova lógica organizacional, em consonância com a reforma sanitária.

Foi a Constituição Federal de 1988 a primeira, no Brasil, a apresentar uma seção sobre saúde. Nela o conceito de saúde é mais amplo, segundo o que foi o discutido na $8^{\text {a }}$ Conferência Nacional de Saúde:

"Em seu sentido mais abrangente, a saúde é o resultado das condições de alimentação, habitação, educação, renda, meio-ambiente, trabalho, transporte, emprego, lazer, liberdade, acesso e posse de terra e acesso a serviços de saúde. É assim, antes de tudo, o resultado das formas de organização social de produção, as quais podem gerar grandes desigualdades nos níveis de vida".

O conceito de saúde deixa bem transparente o direito de cidadania e a relação existente com as políticas sociais e econômicas, compreendendo, então, as ações e serviços de saúde como de relevância pública.

No texto constitucional, observamos avanços que permitiram a continuação da luta política entre os projetos da reforma sanitária e neoliberal.

Na década de 90, foram aprovadas 2 leis: a Lei $8080^{(2)}$, e a Lei $8142^{(3)}$. A Lei Orgânica de Saúde ou Lei 8.080 regulamenta a organização e o funcionamento dos serviços e dispõe sobre as condições para a promoção, proteção e recuperação da saúde. A Lei 8.142 regulamenta a participação popular no SUS e condiciona a locação de recursos financeiros para a saúde à existência e funcionamento do Conselho Municipal de Saúde e dispõe sobre a participação popular na gestão do SUS e sobre as transferências de recursos financeiros para a saúde.

\section{OBJETIVOS}

Relatar a situação das comissões de controle de infecção hospitalar no contexto das políticas de saúde em Santa Catarina. 
A INFECÇÃO HOSPITALAR NO CONTEXTO DAS POLÍTICAS RELATIVAS À SAÚDE NO BRASIL

Em 1980, com o episódio da morte do presidente Tancredo Neves, a infecção hospitalar torna-se popular pelo trabalho realizado pela mídia. É nesse período que começam, então, as denúncias das infecções hospitalares, e seus riscos passam a fazer parte do cotidiano da população brasileira, por meio das manchetes dos jornais.

A infecção hospitalar $(\mathrm{IH})$ representa um dos principais problemas da qualidade da assistência médica, um problema econômico devido à importante incidência e letalidade significativa, assim como os custos diretos, além dos custos indiretos, como aqueles representados pela impossibilidade de retorno ao mercado de trabalho e os custos inatingíveis ou difíceis de se avaliar economicamente, como os distúrbios provocados pela dor, mal-estar, isolamento, enfim, pelo sofrimento experimentado pelo paciente.

A ocorrência da IH amplia o prejuízo, saindo da esfera institucional para o próprio paciente e para a sociedade como um todo.

O controle da infecção hospitalar, além de atender às exigências legais e éticas, tornou-se, também, uma necessidade econômica, principalmente em países como - Brasil, no qual os recursos destinados à saúde são extremamente escassos.

O controle da infecção hospitalar encontra-se entre os requisitos mínimos da proposta de acreditação de hospitais para a América Latina e o Caribe.

"A problemática de infecção hospitalar ocupa posição de destaque na medicina atual, exigindo avaliação epidemiológica atualizada e o desenvolvimento de filosofia e prática de atuação que possa, senão resolver, minorar os resultados adversos da disseminação de doença infecciosa adquirida nos hospitais"(4).

Diante dessa situação, a infecção hospitalar passou a ser vista como um epifenômeno, que serve como importante índice da qualidade da assistência médicohospitalar, e o serviço de prevenção passou a ser considerado programa prioritário de garantia de qualidade, na área de assistência médica.

O assunto foi tomando outras proporções, até que, após algumas atitudes legislativas e normativas governamentais, o Ministério da Saúde (MS) publicou a Portaria $196^{(5)}$, de 1983, que, de forma didática, estabelece critérios para a classificação das infecções hospitalares, obrigando os hospitais a criarem comissão de controle de infecção hospitalar $(\mathrm{CClH})$, como órgão responsável pela determinação de normas, rotinas e vigilância das infecções hospitalares, normatizando aspectos importantes no controle de infecção, não conseguindo, entretanto, o impacto desejado.

O Ministério da Saúde inicia então, cursos de introdução ao controle de IH, em nível nacional, e cria 44 centros de treinamento, visando capacitar os profissionais da área da saúde. No final da década de 80 , formam-se várias associações dos profissionais para estudo e controle das IH: a Associação Paulista de estudos em controle de infecção hospitalar (APECIH), iniciada, como núcleo, em 1982, e transformada em associação em 1987, em São Paulo, a Associação Mineira de estudos em controle de infecção hospitalar (AMECIH), em Minas Gerais, e a Associação Brasileira dos Profissionais em Controle de Infecção Hospitalar (ABIH), fundada em 1987. Essas associações ofereciam cursos básicos e congressos sobre controle de infecção, como, por exemplo, o Curso Internacional de Infecções Hospitalares, financiado pela OPAS/OMS, realizado em Brasília, de 26 a 31 de março de 1984, e o I Congresso Brasileiro sobre Infecção Hospitalar, realizado em São Paulo, em 1989, organizado pela APECIH, etc.

Em 1992, foi promulgada a Portaria $930^{(6)}$, do MS, em substituição à Portaria 196, que regulamenta a vigilância e o controle das infecções hospitalares no país. Essa Portaria determina que todos os hospitais mantenham um programa de controle de infecções hospitalares, com um conjunto de ações desenvolvidas, deliberadas sistematicamente, com vistas à redução máxima possível da incidência e da gravidade das infecções hospitalares.Até 1994, não se conhecia a magnitude dos problemas das infecções hospitalares, lacuna essa que começou a ser preenchida pela iniciativa da Coordenação de Controle de Infecção Hospitalar (COCIN), que idealizou, planejou e coordenou o primeiro estudo brasileiro da magnitude das infecções hospitalares em hospitais terciários $^{(7)}$.

Este estudo contou com uma equipe multidisciplinar de profissionais atuantes em controle de infecção hospitalar $(\mathrm{CIH})$ de todo o país. Para que houvesse uma homogeneização de conceitos relativos à $\mathrm{IH}$, a equipe passou por um treinamento teórico-prático, com o objetivo de que a aplicação do instrumento de coleta de dados e técnica de entrevista tivesse a mesma interpretação. 
O MS realiza, além do estudo, uma pesquisa nacional simultânea, com o objetivo de conhecer e avaliar a qualidade das ações em controle de infecção hospitalar.

Os dados encontrados nesses estudos demonstram os riscos que as infecções hospitalares representam para a sociedade brasileira e ratificam os dados do MS de que as infecções hospitalares têm crescido na razão direta das técnicas invasivas, enquanto o conhecimento dos profissionais de saúde sobre as práticas que controlam infecções não acompanham este desenvolvimento.

Pode-se ressaltar que os dados encontrados deixam evidente que a freqüência média de hospitais executando procedimentos de risco corretamente foi de $42,5 \%$. Os resultados revelam que, dentre os procedimentos das quatro topografias que constituem risco para infecção hospitalar, a menor qualidade e freqüência média de ações corretas foi encontrada nos procedimentos da via circulatória (26,5\%); os procedimentos com a via urinária foram, em média, executados corretamente $(35,0 \%)$ e, destes, a rotinização dos procedimentos foi a menos freqüente $(9,1 \%)^{(7)}$.

Os procedimentos com as vias respiratória tiveram desempenho global (36,9\%), também insuficiente para gerar qualidade e evitar infecção hospitalar, a partir de cuidados preventivos durante a instalação e manutenção dos procedimentos.

A freqüência média de hospitais executando procedimentos invasivos corretamente foi de $51,5 \% \mathrm{em}$ procedimentos cirúrgicos, o uso de antibiótico profilático dentro de critérios adequados, $14,1 \%$, a troca de luvas durante $o$ ato cirúrgico condicionada ao tempo de duração cirúrgica, 22,2\%, e a preservação do ambiente cirúrgico, $30,3 \%$. Foram os itens menos encontrados na prática de prevenção das infecções cirúrgicas dos hospitais estudados $^{(7)}$.

O MS divulga novos dados em 1995, os quais apontam uma incidência nacional média de $\mathrm{IH}$ de $15,5 \%$, com variância de 14,8\% a 16,3\% (com limite de confiança de $95 \%$, numa razão de 1,18 infecções por paciente. A avaliação da qualidade das ações de $\mathrm{ClH}$ foi realizada com o objetivo de avaliar o desenvolvimento das ações de assistência direta hospitalar e orientar plano de diretrizes brasileiras para essa área. Os resultados mostraram que a média de pontos dos hospitais foi de um terço do máximo esperado ( $35 \% \pm 17 \%)$. O desempenho e as medidas de prevenção das $\mathrm{IH}$, a partir de procedimentos de risco para adquirí-las, é de $42,5 \%$, conforme resultado ${ }^{(7)}$.
Em 12 de maio de 1998, o MS revoga a portaria 930 e expede a Portaria $2616^{(8)}$ que, no âmbito nacional, rege o controle de infecção hospitalar e mantém o Programa de Controle de Infecção hospitalar, sendo que os membros do Serviço de Controle de Infecção Hospitalar (SCIH) exercem a função de membros executores, e os membros da $\mathrm{CCIH}$ passam a ser membros consultores.

\section{A INFECÇÃO HOSPITALAR NO CONTEXTO DAS POLÍTICAS RELATIVAS À SAÚDE EM SANTA CATARINA}

Quando houve a implantação do Programa Nacional de Controle de Infecção Hospitalar, pelo MS, em 1983, Santa Catarina contava com poucos hospitais desenvolvendo ações nessa área. Somente a partir de 1985, com a criação do centro de treinamento de recursos humanos para controle de infecções hospitalares, no Hospital Infantil Joana de Gusmão, teve início um processo de conscientização dos profissionais para a existência do problema, embora atuando de forma voluntária e sem uma estrutura adequada para tal empreitada, o qual passou a ser um referencial no que dizia respeito à organização das CCIH. Esse Centro funcionou até 1991 e foi responsável pela formação inicial em controle de infecção hospitalar de, aproximadamente, 200 profissionais de nível superior, provenientes dos hospitais de grande e médio porte do Estado.

Apesar de não ter ocorrido avaliação do aproveitamento dos egressos em relação ao aprendizado, estima-se que a maioria não conseguiu aplicar os conhecimentos adquiridos, pois as instituições não estavam preparadas, nem motivadas a investirem na contratação de recursos humanos para a finalidade específica.

Com a mudança de Governo e término do Centro de Treinamento, assume a Diretoria de Assuntos Hospitalares que realizou um levantamento por meio de questionário dirigido às Direções dos Hospitais e concluiu que apenas $7 \%$ ou 14, de um universo de 207 hospitais, possuía $\mathrm{CClH}$. Com esse resultado, abaixo da estimativa do País, que é de $10 \%$, partiu para uma ação mais efetiva e propôs a criação da Gerência de Controle de Infecção Hospitalar, em sua estrutura administrativa.

Em 1991, o Estado assumiu a responsabilidade do Programa, criando uma Unidade de Controle de 
Infecção Hospitalar para atuar na prevenção e controle das infecções hospitalares, desempenhando importante papel.

O Programa foi criado por meio da Portaria 01/92/ SES/SC ${ }^{(9)}$, em 15 de maio de 1992, sendo aprovado em 16 de julho de 1992, pela Assembléia Legislativa, incluindo a Gerência de Controle de Infecção Hospitalar no organograma da Secretaria de Estado da Saúde (SES), como um órgão de assessoramento técnico e administrativo da Diretoria de Assuntos Hospitalares, voltado para a coordenação e o planejamento das ações de prevenção e controle das Infecções Hospitalares, em hospitais da rede pública e privada de Santa Catarina. Desde então, vem buscando estimular a criação das $\mathrm{CCIH}$, de maneira sistemática, utilizando recursos e estratégias as mais diversas, como o envio de ofícios circulares, alertando para as exigências legais e implicações jurídicas possíveis, fornecendo informações técnicas e científicas que venham ao encontro das necessidades dos profissionais de saúde, na execução das atividades diárias de prevenção e controle das infecções hospitalares.

A receptividade a essas ações foi favorável, tanto que as Comissões de Controle de Infecção foram implantadas em 90 hospitais de Santa Catarina o que representou um aumento significativo (7\% em 1992 para 43\% em 1993) de instituições engajadas no Programa.

Dentre os compromissos assumidos pela Gerência de Controle de Infecção Hospitalar, uma das metas era a implantação de comissão de controle de infecção hospitalar, em $50 \%$ dos hospitais de Santa Catarina, até dezembro de 1994.

O Programa Estadual de Controle de Infecção Hospitalar teve como preocupação buscar subsídios para motivar os profissionais atuantes nas comissões, para que estes obtivessem maior receptividade na execução dessas ações, sendo, então, criado o Programa de Acreditação em Controle de Infecção Hospitalar em Santa Catarina, em 1994.

A perseguição dessa meta previa a utilização de várias estratégias, incluindo a publicação de um manual, que buscou facilitar e organizar os procedimentos administrativos necessários para a efetivação do cadastro das $\mathrm{CCIH}$, nesse Órgão.

Pela Gerência de Controle de Infecção Hospitalar foram implantados vários Projetos em 1992, que continuam até os dias atuais, como o Disk \& Saiba Infecção Hospitalar, que serve como um instrumento de apoio técnico aos profissionais de saúde da área hospitalar, principalmente no que se refere à organização e atuação das $\mathrm{CClH}$. Por meio do telefone e fax, o usuário formula as suas dúvidas, é preenchido um formulário, e as respostas são encaminhadas via correio. O Programa de Acreditação em Controle de Infecção Hospitalar foi criado a partir de estudo, em parceria com a Coordenação de Controle de Infecção Hospitalar do Ministério da Saúde, adaptado aos moldes do "Manual de Acreditação dos Hospitais para América Latina e Caribe", da Organização Panamericana da Saúde, que constitui a adoção de padrões mínimos de avaliação do efetivo cumprimento das determinações da Portaria 930 e, atualmente, a Portaria 2616, de 1998.

Em 1998, o Programa de Controle de Infecção Hospitalar de Santa Catarina (SES) divulga os dados por meio do caderno "Manual de Orientações", colocando que, dos 128 hospitais existentes no Estado, 75\% apresentam $\mathrm{CClH}$ formalmente constituída e, desses, apenas 33\% encaminham boletins epidemiológicos ao Programa Estadual de Controle de Infecção Hospitalar $(\mathrm{PECIH})$ da SES. Há um desconhecimento da SES da metodologia utilizada pelos hospitais para coleta de dados, inexistência de testes estatísticos estabelecendo os níveis de confiabilidade do instrumento utilizado pela própria SES, pouco preparo dos profissionais atuantes em CCIH e não explicitação da SES dos critérios utilizados para calcular as taxas.

Atualmente já estão implantadas 177 Comissões de Controle de Infecção Hospitalar, em Santa Catarina, o que representa $77 \%$ do total de hospitais.

A Coordenação Nacional gerencia o programa no âmbito nacional; define as suas diretrizes; fornece condições aos Estados e os incentiva para o desenvolvimento do Programa; normatiza e coordena, no âmbito nacional, as ações do Programa desenvolvidas pelo Sistema Único de Saúde (SUS). O Estado, baseado nas diretrizes nacionais, mantém a Coordenação Estadual que desenvolve uma atuação semelhante à federal, com uma diferença - dá enfoque aos municípios. A Coordenação Municipal deveria ser a instância mais operacional dessa rede hierárquica, porém, no momento atual do Programa, a descentralização das ações ainda não se deu no âmbito dos municípios - grande meta a ser atingida pelo Brasil.

Pela organização dos programas, os organismos de gestão estadual e municipal do SUS deverão viabilizar estrutura técnico-operacional que assegure o cumprimento dos artigos $1^{\circ}, 2^{\circ}$ e $3^{\circ}$, do Decreto $n^{\circ} 77.052^{(10)}$, de 19 de 
janeiro de 1976, bem como o que dispõem os incisos VII, XI e XII, do artigo 17, os incisos III, VII, XI e XII, do artigo 18, e o artigo 19, da Lei oㅡ 8.080, de 19 de setembro de 1990.

Os últimos dados estatísticos do Programa de Acreditação Hospitalar de Santa Catarina mostram que, em 2000, 08 hospitais eram acreditados em todo o Estado, sendo que, desses 2 perderam o título em 2001, por não alcançarem a pontuação mínima de $80 \%$ na avaliação. Atualmente, existem 16 hospitais acreditados em infecção hospitalar, no Estado, e 30 hospitais em fase preparatória para avaliação da acreditação.

\section{CONSIDERAÇÕES}

Em texto editado em 1992, por consultores da Organização Pan Americana da Saúde (OPAS), o Controle da infecção hospitalar já se encontrava entre os requisitos mínimos da proposta de Acreditação de Hospitais para a América Latina e o Caribe. Dentre os fundamentos da garantia de Qualidade para fins de acreditação hospitalar, tais direitos são explicitados nos seguintes termos:

"O paciente tem o direito de participar, razoavelmente informado, nas decisões relacionadas com seu tratamento de saúde. Até onde seja possível, isso deve ser baseado em uma explicação clara e concisa de sua condição e de todos os procedimentos técnicos implícitos, incluindo a possibilidade de qualquer tipo de risco de morte ou de reações sérias, de problemas relacionados com sua recuperação e sua possível alta satisfatória. O paciente não deverá estar sujeito a nenhum procedimento sem seu consentimento voluntário, competente ou o consentimento do seu representante legal autorizado. Quando existirem alternativas médicas significativas para seu tratamento, estas também deverão ser informadas ao paciente".

\section{REFERÊNCIAS BIBLIOGRÁFICAS}

1. Constituição da República Federativa do Brasil. Diário Oficial da União 198805 setembro: Seção A; 191.

2. Ministério da Saúde (BR). Lei Orgânica 8080. Dispõe sobre as condições para a promoção, proteção e recuperação da saúde, a organização e o funcionamento dos serviços correspondentes e dá outras providências. Diário Oficial da União 199019 setembro.

3. Ministério da Saúde (BR). Lei Orgânica 8142. Dispõe sobre a participação da comunidade na gestão do Sistema Único de Saúde (SUS) e sobre as transferências intergovernamentais de recursos financeiros na área da saúde e dá outras providências. Diário Oficial da União 199019 setembro.
Dentro desse contexto, surge o Programa Brasileiro de Acreditação hospitalar que, após teste piloto para sua avaliação, permitiu a inclusão de excelentes sugestões de consultores técnicos e representantes das instituições que hoje trabalham com acreditação. O Ministério da Saúde, responsável pela determinação de políticas globais de saúde, poderá estabelecer como meta que, dentro de um determinado período de tempo, o Programa de Acreditação Hospitalar seja implantado em todos os hospitais do país e, para tanto, deverá ter como base duas importantes variáveis: avaliação dos padrões de referência desejáveis, construídos por peritos e previamente divulgados e, os indicadores, isto é, os instrumentos que o avaliador/visitador usará para constatar se os padrões foram observados ou estão presentes na instituição.

Finalmente, podemos argumentar que a questão moderna da qualidade de bens e serviços tem, inegavelmente, vínculos históricos com a chamada polícia sanitária e, em sentido mais amplo, com os problemas que cercam a proteção ao consumidor. "O que, hoje, é uma opção de oferecer o melhor, foi, ontem, uma opção de oferecer o que elimina ou diminui o risco de dano à saúde da população.

O nó górdio de todas as divergências é, como se sabe, determinar o quanto é possível coadunar um elemento econômico e político, a liberdade de mercado, com um elemento moral, o resguardo da saúde e dos demais interesses do consumidor"(11).

O ponto crítico da questão encontra-se na disjunção verificada quando se diz que a saúde não tem preço (o que todos reconhecem), mas tem custos (o que é dramaticamente vivenciado pelos provedores de serviços). É o que se descobre ao considerar o processo de produção de serviços de saúde sob a óptica da gestão de recursos escassos dirigidos para a satisfação de necessidades aparentemente inesgotáveis ${ }^{(11)}$.

4. Laforce FM. The control of infections in hospitals: 150 to 1950. In: Wenzel RP. Prevention and Control of Nosocomial Infections. Baltimore: Williams \& Wilkins; 1987. p.1-12. 5. Ministério da Saúde (BR). Portaria no 196. Dispõe sobre a obrigatoriedade de todos os hospitais do País manterem a Comissão de Controle de Infecção Hospitalar (CCIH). Diário Oficial da União 198324 junho.

6. Ministério da Saúde (BR). Portaria no 930. Dispõe sobre controle de prevenções de infecções hospitalares. Diário Oficial da União 199227 agosto.

7. Prade SS, Felix J, Mendes A, Gadelha MZ, Pereira M. Estudo Brasileiro da Magnitude das Infecções Hospitalares em hospitais terciários. Rev Controle de Infecção Hospitalar 1995 mar/jul; 2:11-24. 
8. Ministério da Saúde (BR). Portaria no 2616. Dispõe sobre a regulamentação das ações de controle de infecção hospitalar no país. Diário Oficial da União 199812 maio.

9. Secretaria de Estado da Saúde (SC). Diretoria de Assuntos Hospitalares Resolução 01/92/SES/SC, Resolve criar o Programa Estadual de Controle de Infecção Hospitalar em Santa Catarina. Diário Oficial do Estado 199215 de maio.

10. Ministério da Saúde (BR). Decreto 77052. Dispõe sobre a fiscalização sanitária das condições de exercício de profissões e ocupações técnicas e auxiliares, relacionadas diretamente com a saúde. Diário Oficial da União 197619 janeiro.

11. Nogueira RP. Perspectivas da qualidade em saúde. Rio de Janeiro: Qualitymark; 1994. 